Computer Science \& Engineering: An International Journal (CSEIJ), Vol.2, No.2, April 2012

\title{
Automated Detection of White MATTER LESIONS IN MRI BRAIN IMAGES USING SPATIO- FUZZY AND SPATIO-PossibILISTIC ClusteriNg MODELS
}

\author{
M.Anitha ${ }^{1}$, Prof.P.Tamije Selvy ${ }^{2}$ and Dr.V.Palanisamy ${ }^{3}$ \\ ${ }^{1}$ PG Student, ${ }^{2}$ Assistant Professor, ${ }^{3}$ Principal \\ ${ }^{1,2}$ Department of Computer Science and Engineering, Sri Krishna College of Technology, \\ Coimbatore, India \\ ${ }^{1}$ anitha_murugaiyan@yahoo.com, ${ }^{2}$ tamijeselvy@gmail.com \\ ${ }^{3}$ Info Institute of Engineering, Coimbatore, India \\ ${ }^{3}$ vpalanisamy@gmail. com
}

\begin{abstract}
White Matter Lesions (WMLs) are small areas of dead cells found in parts of the brain. In general, it is difficult for medical experts to accurately quantify the WMLs due to decreased contrast between White Matter (WM) and Grey Matter (GM). The aim of this paper is to automatically detect the White Matter Lesions which is present in the brains of elderly people. WML detection process includes the following stages: 1. Image preprocessing, 2. Clustering (Fuzzy c-means clustering (FCM), Geostatistical Possibilistic clustering (GPC) and Geostatistical Fuzzy clustering (GFCM)). The proposed system is tested on a database of 208 MRI images. GFCM yields high sensitivity of $90 \%$, specificity of $94 \%$ and overall accuracy of $95 \%$ over FCM and GPC. The detection results reveal that GFCM better localizes the large regions of lesions and gives less false positive rate when compared to FCM and GPC which captures the largest loads of WMLs only in the upper ventral horns of the brain.
\end{abstract}

\section{KEYWORDS}

Fuzzy Clustering, Geostatistics, Possibilistic Clustering \& White Matter Changes

\section{INTRODUCTION}

Medical imaging is the technique used to create images of the human body for clinical or medical science that produce images of the internal aspect of the body. Magnetic Resonance Imaging (MRI) is one of the medical imaging techniques. MRI of brain is highly sensitive for detecting all forms of White Matter abnormalities. Non-specific changes in the White Matter appear frequently on MRI in elderly patients presenting with either stroke or cognitive impairment. In general, human brain consists of main components namely, White Matter (WM), Grey Matter (GM) as shown in Fig. 1. Neuronal tissue containing mainly long, myelinated axons is known as White Matter. Closely packed neuron cell bodies form the Grey Matter. Grey Matter is in grey color because of the grey nuclei that comprises the cells. Myelin is responsible for the white appearance of White Matter. White Matter Lesions (WMLs) are commonly found in patients with Multiple Sclerosis (MS), Cerebrovascular Disease (CVD), stroke, and other neurological disorders. It is DOI : $10.5121 /$ cseij.2012.2201 
believed that the total volume of the lesions and their progression relate to the aging process as well as disease process. Therefore, quantification of White Matter Lesions is very important in understanding the aging process and diagnosis and assessment of these diseases.

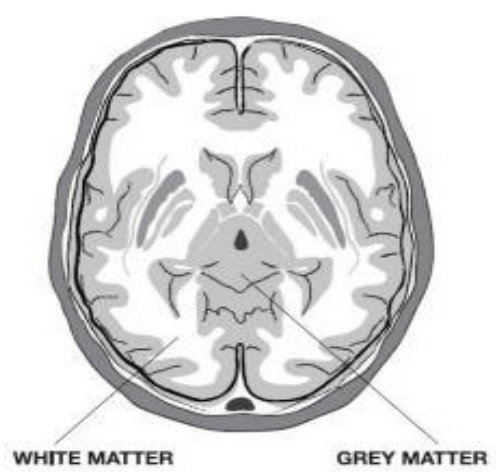

Figure 1. WM and GM of brain

\subsection{Automated Quantification of WML}

Non-specific changes in the White Matter appear frequently on CT and MRI in elderly patients presenting with either stroke or cognitive impairment, but are also commonly seen in healthy elderly individuals. Evaluation of WMLs in MRI is conventionally performed using skill and knowledge of experts [2]. This manual assessment on WML results in different ratings [1], [3], which make it non reproducible and difficult for a general agreement. Manual assessment of WM lesions is not only time consuming but also shows high inconsistency among human raters. To overcome this drawback of inaccurate prediction, clustering models like Fuzzy-set, Possibilistic and Geostatistic frameworks are proposed for automated detection of White Matter changes. The proposed clustering models are derived by extending the objective functions of FCM and Possibilistic approach with a Geostatistical (spatial) model.

\section{RELATED WORKS}

Anbeek et al. [4] proposed $k$-nearest neighbors algorithm $(k-\mathrm{NN})$ for automatic segmentation of WMLs. This is a supervised learning method and used the information from T1-weighted, inversion recovery (IR), proton density-weighted (PD), T2-weighted, and fluid attenuation IR (FLAIR) scans in order to estimate the probability of voxels. T1-weighted scans are a standard basic scan, in particular differentiating fat from water - with water darker and fat brighter. This is one of the basic types of MR contrast and is a commonly run clinical scan. $T 2$ - weighted scans are another basic type. Like the $T 1$-weighted scan, fat is differentiated from water - but in this case fat shows darker, and water lighter. For example, in the case of cerebral and spinal study, the CSF (cerebrospinal fluid) will be lighter in T2-weighted images. By combining the results of these techniques, binary segmentation results are obtained from the selected threshold values, and therefore the relation between an optimal threshold and lesion volume was separately chosen for each patient.

A probability mixture model and the Bayesian classifier was used by Khayati et al. [5] inorder to extract normal tissue, abnormal tissue and cerebrospinal fluid (CSF) which serves primary purpose like buoyancy, protection and chemical stability. Normal tissue refers to White Matter and Grey Matter of brain whereas abnormal tissue refers to lesions of brain in FLAIR-MR images. This method does not focus on the lesions of small size or irregular shape. 
Lao et al. [6] proposed an approach for segmenting WML in which support-vector machine (SVM) classifier was used in order to classify new scans, and post processing analysis was carried out to eliminate false positives. The strength of SVM-based classifiers is the ability to separate overlapping features, but selecting effective features for classifying a particular difficult problem is one of the key issues in pattern classification that should be first identified, where the results are based on only expert-defined information. This method is less accurate. Therefore WML image intensities cannot be visually distinguished.

Lesions are irregular voxels that do not belong to GM, WM and CSF and can be classified as outliers in grey and White Matter regions. This method was proposed by Seghier et al. [7]. After combining the segmentation and normalization of images, fuzzy clustering was applied to identify outlier voxels as lesions in normalized grey and White Matter segments. Spatial smoothing was done using Gaussian kernel, which affect the sensitivity and specificity of the method.

Hernandez et al. [8] presented a multispectral MRI approach for segmenting normal and abnormal brain tissue. The procedure was carried out by combining pairs of different MRI sequences and modulated them in the red-green color space to enhance the tissue discrimination. Fully automated method for CSF, GM and WM segmentation based on multimodal MRI data is optimized and extended with WML segmentation was proposed by R. de Boer, H. A. Vrooman, F. van der Lijn [9].

\section{Proposed Scheme}

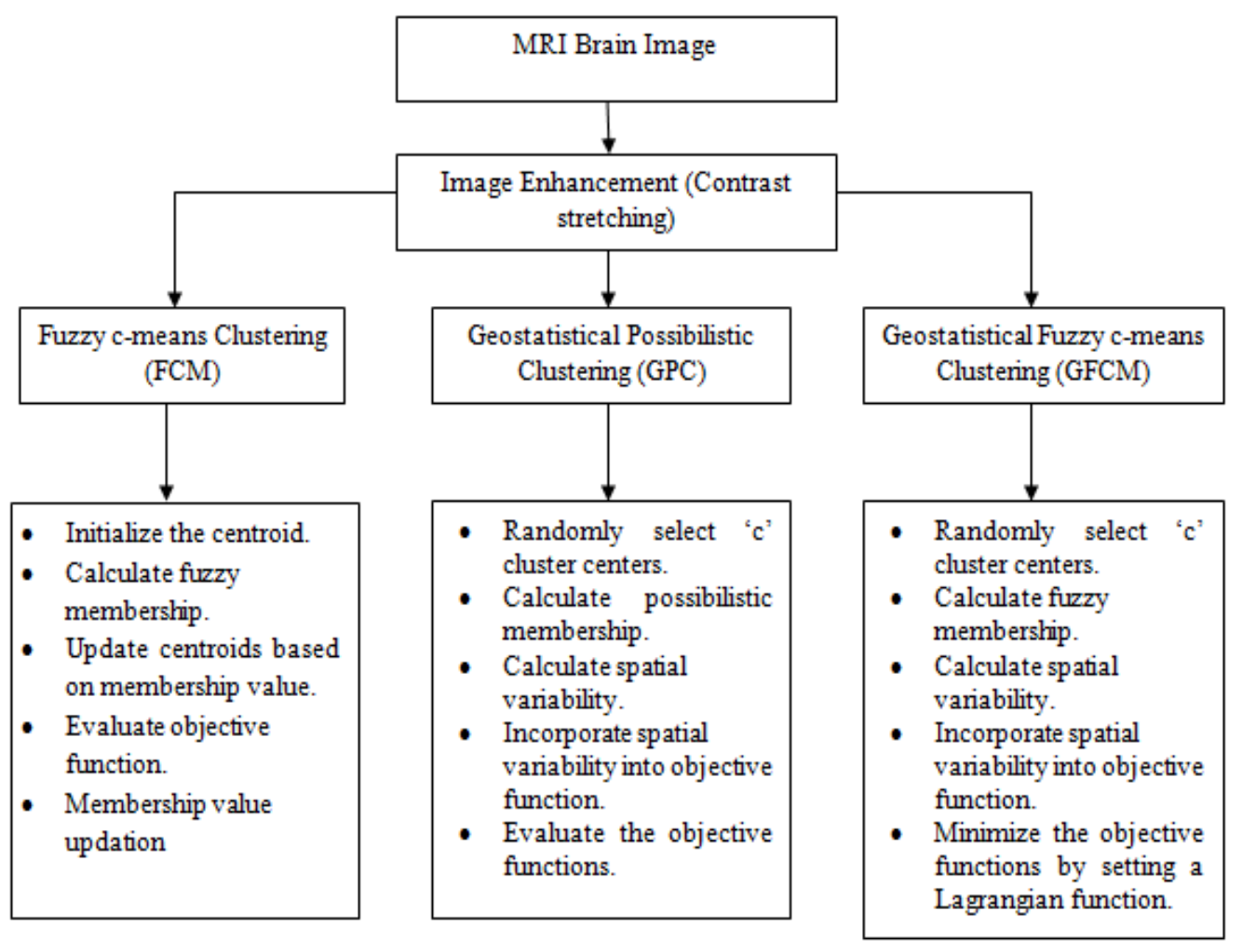

Figure 2. Overall process of the system 
This paper mainly focuses on automated detection of White Matter Lesions of brain using fast and efficient clustering algorithms. The goal of clustering a medical image is to simplify the representation of an image into a meaningful image and makes it easier to analyze. As a first step, MRI brain image is pre-processed using Contrast Stretching technique which is one of the efficient image enhancement techniques. The pre-processed image is subjected to clustering. The clustering algorithms include Fuzzy c-means Clustering (FCM), Geostatistical Possibilistic Clustering (GPC) and Geostatistical Fuzzy Clustering Model (GFCM). However clustering techniques are sensitive to initialization and are easily trapped in local optima. Fig.2 represents overall process of automatic detection of WMLs of brain. Since MS lesions present different characteristics from lesions in elderly individuals there are many clustering models to determine the accuracy but those methods are not directly applicable to predict the accurate lesions because of the decreased contrast between White Matter and Grey Matter in elderly people. The proposed clustering models are derived by extending the objective functions of FCM and Possibilistic clustering with a Geostatistical (Spatial) model. These algorithms are applied to real magnetic resonance images and is shown to be more robust to noise and other artifacts than competing approaches.

\subsection{Image Enhancement (Pre-processing)}

The procedure done before processing by correcting image from different errors is preprocessing. Image enhancement is one of the image preprocessing techniques. The aim of image enhancement is to improve the interpretability or perception of information in images for human viewers, or to provide 'better' input for other automated image processing techniques. It consists of collection of techniques that seek to improve the visual appearance of an image or to convert the image to a form better suited for analysis by a human or machine [15]. Contrast stretching is the image enhancement technique that is commonly used for medical images. Contrast stretching process plays an important role in enhancing the quality and contrast of medical images. Different types of contrast stretching techniques include local contrast stretching, global contrast stretching, partial contrast stretching, bright and dark contrast stretching. Among these techniques, bright contrast stretching is imposed on the brain image.

\subsection{Fuzzy C-Means Clustering (FCM)}

Fuzzy c-means has been a very important tool for image processing in clustering objects in an image [16]. Fuzzy c-means (FCM) clustering is an unsupervised method derived from fuzzy logic that is suitable for solving multiclass and ambiguous clustering problems. Fuzzy c-means (FCM) clustering is an unsupervised technique that has been successfully applied to feature analysis, clustering, and classifier designs in fields such as astronomy, geology, medical imaging, target recognition, and image segmentation. An image can be represented in various feature spaces, and the FCM algorithm classifies the image by grouping similar data points in the feature space into clusters. This clustering is achieved by iteratively minimizing a cost function that is dependent on the distance of the pixels to the cluster centers in the feature domain. There are many acceleration techniques for FCM; there are very large data versions of FCM that utilize both progressive sampling and distributed clustering; there are many techniques that use FCM clustering to build fuzzy rule bases for fuzzy systems design; and there are numerous applications of FCM in virtually every major application area of clustering. FCM clustering algorithm is used to calculate the minimization of the fuzzy objective function [11]. It works by assigning membership to each data point corresponding to each cluster center on the basis of distance between the cluster center and the data point. 
The fuzzy clustering of objects is described by a fuzzy matrix $\mu$, with $\mathrm{n}$ rows and c columns in which $n$ is the number of data objects and $c$ is the number of clusters. $\mu_{\mathrm{ij}}$, the element in the ith row and jth column in $\mu$, indicates the degree of association or membership function of the $i^{\text {th }}$ object with the $j^{\text {th }}$ cluster. The characters of $\mu$ are as follows:

$$
\begin{aligned}
& \mu_{i j}[0,1], \quad \quad i=1,2 \ldots, n, j=1,2 \ldots, c \\
& \text { where, } \quad \sum_{j=1}^{c} \mu_{i j}=1, \quad i=1,2 \ldots, n \\
& 0<\sum \mu_{\mathrm{ij}}<\mathrm{n}, \quad \quad \mathrm{j}=1,2 \ldots \mathrm{c}
\end{aligned}
$$

More the data is near to the cluster center more is its membership towards the particular cluster center. Clearly, summation of membership of each data point should be equal to one. Its advantages include a straightforward implementation, fairly robust behaviour, applicability to multichannel data. Main objective of fuzzy c-means algorithm is to minimize the objective function.

$$
\mathrm{J}(\mathrm{U}, \mathrm{V})=\sum_{\mathrm{i}=1}^{\mathrm{n}} \sum_{\mathrm{j}=1}^{\mathrm{c}}\left(\mu_{\mathrm{ii}}\right)^{\mathrm{m}}\left\|x_{\mathrm{i}}-v_{\mathrm{i}}\right\|^{2}
$$

where, $m(m>1)$ is a scalar termed the weighting exponent and controls the fuzziness of the resulting clusters and ' ||$x_{i}-v_{j}||^{\prime}$ is the Euclidean distance between $\mathrm{i}^{\text {th }}$ data and $\mathrm{j}^{\text {th }}$ cluster center. The $\mathrm{z}_{\mathrm{j}}$, centroid of the jth cluster, is obtained using Eq. (5).

$$
Z_{j}=\sum_{i=1}^{n}\left(\mu_{i j}\right)^{m} x_{i} / \sum_{i=1}^{n}\left(\mu_{i j}\right)^{m}
$$

\subsubsection{FCM Algorithm}

S1 Randomly select ' $c$ ' cluster centers.

S2 Compute the Euclidean distance, $\left\|x_{\mathrm{i}}-v_{\mathrm{j}}\right\|$.

S3 Calculate the fuzzy membership according to the constraints of Eq. (1), (2) and (3).

S4 Calculate the fuzzy center according to Eq. (5).

S5 Repeat steps 2) and 3) until the minimum ' $J$ ' value is incorporated as in Eq. (4).

\subsection{Geostatistical Possibilistic Clustering (GPC)}

Although FCM is a very useful clustering method, its memberships do not always correspond well to the degree of belonging of the data [17], and may be inaccurate in a noisy environment. To improve this weakness of FCM and to produce memberships that have a good explanation for the degree of belonging for the data, Possibilistic approach was proposed. It is a variation over fuzzy clustering where the membership to clusters can be seen as a degree of typicality membership matrix $\mathrm{U}, u_{i h} \in[0,1]$. Possibilistic clustering algorithms prove the fact that it can be applied for one cluster at a time. 
$J_{G P}(U, v)=\sum_{i=1}^{N} \sum_{j=1}^{c}\left(u_{i j}\right)^{m}\left[d\left(x_{i}, v_{j}\right)\right]^{2}+\sum_{j=1}^{N} 1 /\left(e_{j}\right)^{2} \sum_{i=1}^{c}\left(1-u_{i j}\right)^{m}$

where, $\left(e_{j}\right)^{2}$ is the kriging (geostatistical) variance of estimating $\mathrm{v}_{\mathrm{j}}$ using $\mathrm{x} i, i=1 \ldots \mathrm{N}-1$.

\subsubsection{GPC Algorithm}

S1 Randomly select ' $c$ ' cluster centers according to Eq. (5).

S2 Calculate the possibilistic membership.

S3 Calculate the spatial variability.

S4 Incorporate spatial variability into objective functions as in Eq. (6).

S5 Minimize the objective functions (a small value of difference) then stop.

\subsection{Geostatistical Fuzzy c-means Clustering (GFCM)}

The fuzzy $C$-means objective function is generalized to include a spatial penalty on the membership functions. The fuzzy $C$-means algorithm (FCM) has been utilized in a wide variety of image processing applications such as medical imaging and remote sensing. Its advantages include a straightforward implementation, fairly robust behavior, applicability to multichannel data, and the ability to model uncertainty within the data. A major disadvantage of its use in imaging applications is that FCM does not incorporate information about spatial context, causing it to be sensitive to noise and other imaging artifacts. Therefore geostatistical fuzzy clustering is proposed. The advantages of the new method are the following: (1) it yields regions more homogeneous than those of other methods, (2) it removes noisy spots, and (3) it is less sensitive to noise than other techniques. It is derived by extending the into the FCM objective function. Clustering is a two-pass process at each iteration [18]. The first pass is the same as that in standard FCM to calculate the membership function in the spectral domain. In the second pass, the membership information of each pixel is mapped to the spatial domain, and the spatial function is computed from that. The FCM iteration proceeds with the new membership that is incorporated with the spatial function. The iteration is stopped when the maximum difference between two cluster centers at two successive iterations is less than a threshold. A distinctive observation of the incorporation of the geostatistical modeling into the fuzzy clustering is that it is able to accurately detect the WMLs as the regions of interest of an elderly population and provides the bidirectional association between depression and vascular disease. The main aim of is to minimize the objective function, where kriging variance is incorporated It is a derived function.

$\mathrm{J}_{\mathrm{GF}}(\mathrm{U}, v)=\sum_{\mathrm{i}=1 \mathrm{j}=1}^{\mathrm{N}} \sum_{\mathrm{ij}}^{\mathrm{c}}\left(\mathrm{u}_{\mathrm{ij}}\right)^{\mathrm{m}}\left[\mathrm{d}\left(x_{i}, v \mathrm{j}\right)\right]^{2}+\sum_{\mathrm{i}=1}^{\mathrm{N}}\left(\mathrm{u}_{\mathrm{ij}}\right)^{\mathrm{m}} \sum_{\mathrm{j}=1}^{\mathrm{c}}\left(\mathrm{e}_{\mathrm{j}}\right)^{2}-\sum_{\mathrm{i}=1}^{\mathrm{n}} \lambda_{\mathrm{i}}\left(\sum_{\mathrm{j}=1}^{\mathrm{c}} \mathrm{u}_{\mathrm{ij}}-1\right)$

\subsubsection{GFCM Algorithm}

S1 Randomly select ' $c$ ' cluster centers.

S2 Calculate the fuzzy membership.

S3 Calculate the spatial variability.

S4 Incorporate spatial variability into objective function as in Eq. (7).

S5 Minimize the objective functions by setting a Lagrangian function. 


\section{EXPERIMENTAL RESULTS}

The performance of WML quantification is evaluated using clustering algorithms. When the image is pre-processed, contrast of the image is enhanced. The resulting enhanced image is clustered using the effective clustering algorithms. Fig. 3 represents the input image for WML detection. In order to increase robustness, the noisy medical image is pre-processed.

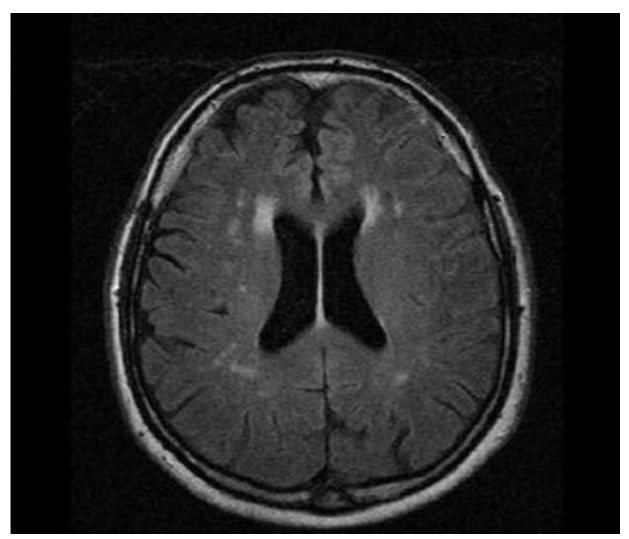

Figure 3. MRI of FLAIR image

Fig. 4 depicts the pre-processed image. Bright contrast stretching, which is one of the image enhancement (pre-processing) techniques is applied. After pre-processing the enhanced image is subjected to clustering. Three clustering models are proposed to provide accurate results. The algorithms include (1) FCM, (2) GPC and (3) GFCM.

The first method proposed is fuzzy-c means clustering. When FCM is applied, numerous small regions around the lobes (ventral, parietal, occipital and temporal) are falsely detected as shown in Fig. 5. Fig. 6 shows the extracted WMLs which are detected using Geostatistical Possibilistic Clustering. GPC could better capture the largest loads of WMLs only in the upper ventral horns and it failed to detect other smaller regions of the White Matter changes. When GFCM is applied, the regions containing White Matter Lesions are accurately quantified as shown in Fig.7. This method provides the best results when compared to FCM and GPC, and provides accuracy of $95 \%$ as it incorporates the spatial information.

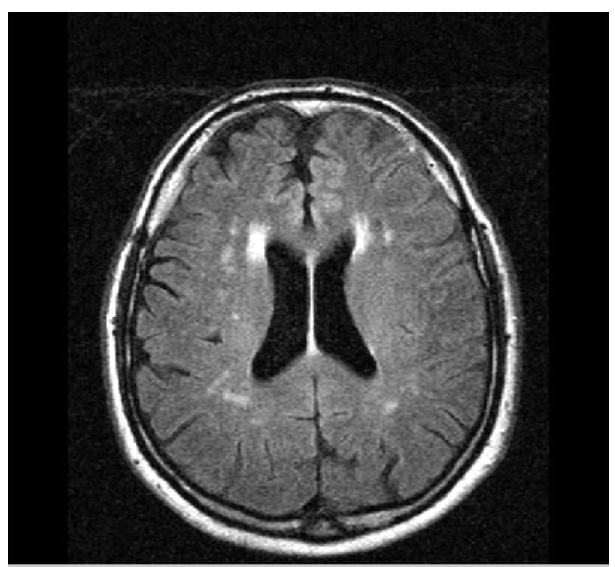

Figure 4. Enhanced image

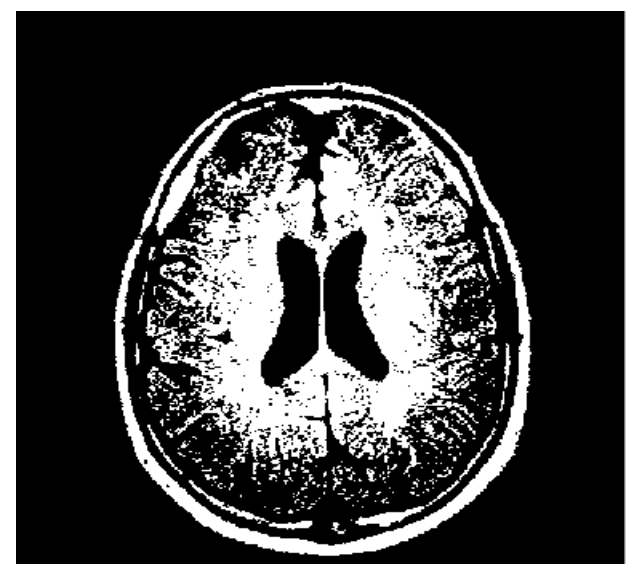

Figure 5. WML detection using FCM 


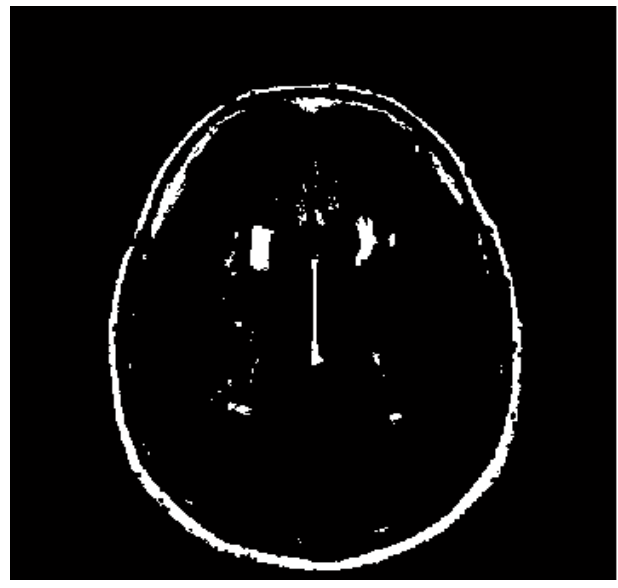

Figure 6. WML detection using GPC

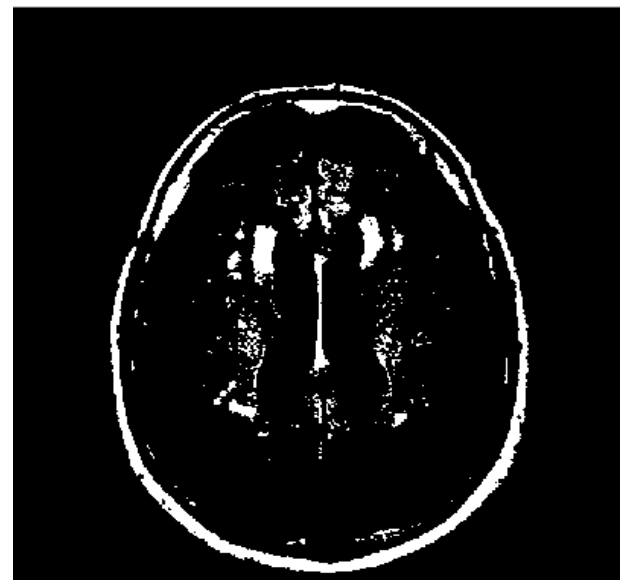

Figure 7. WML detection using GFCM

All scans obtained from different image clustering models are manually ranked based on values in Table I. FCM, GPC and GFCM clustering methods are applied on a dataset of 208 images and ranking is done in terms of under detected, over detected, properly detected as shown in Fig. 8. The number of images detected properly in GFCM is comparatively high than FCM and GPC.

Table I

WML Detection Rates (\%) of FCM, GPC and GFCM

\begin{tabular}{|c|c|c|c|}
\hline Model & Over Detected & Under detected & Properly detected \\
\hline FCM & 121 & 120 & 144 \\
\hline GPC & 120 & 112 & 165 \\
\hline GFCM & 74 & 92 & 193 \\
\hline
\end{tabular}

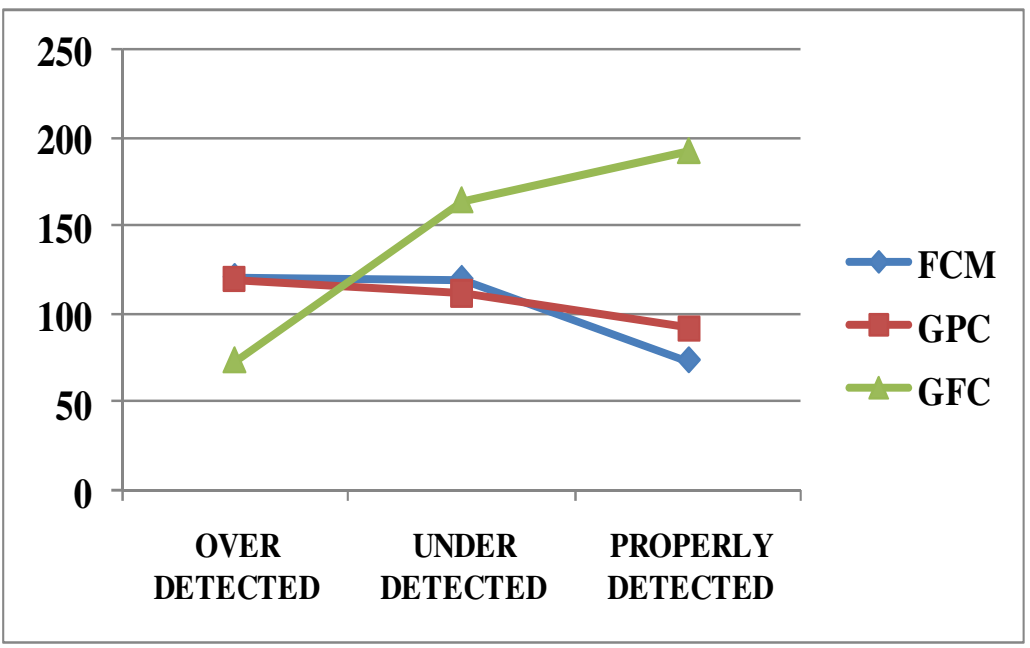

Figure 8. WML detection rates of FCM, GPC and GFCM 
In this paper, clustering algorithms are evaluated in terms of sensitivity $(S e)$, specificity $(S p)$ and accuracy $(A c c)$. Taking Table II into account the metrics are defined as

$$
\begin{aligned}
S e & =\frac{T P}{T P+F N} \\
S p & =\frac{T P}{T N+F P} \\
A c c= & \frac{T P+T N}{T P+F N+T N+F P}
\end{aligned}
$$

where, TP-True Positive, FN-False Negative, TN-True Negative, FP-False Positive. The performance results show that GFCM provides sensitivity ( $\mathrm{Se}$ ) of $90 \%$, specificity $(\mathrm{Sp})$ of $94 \%$ and overall accuracy $(A c c)$ of $95 \%$ when compared to FCM and GPC.

Table II

Performance results of FCM, GPC, GFCM and hybrid methods on FLAIR image database

\begin{tabular}{|c|c|c|c|}
\hline Model & Sensitivity (\%) & Specificity (\%) & Accuracy (\%) \\
\hline FCM & 55 & 62 & 60 \\
\hline GPC & 76 & 78 & 74 \\
\hline GFCM & 90 & 94 & 95 \\
\hline
\end{tabular}

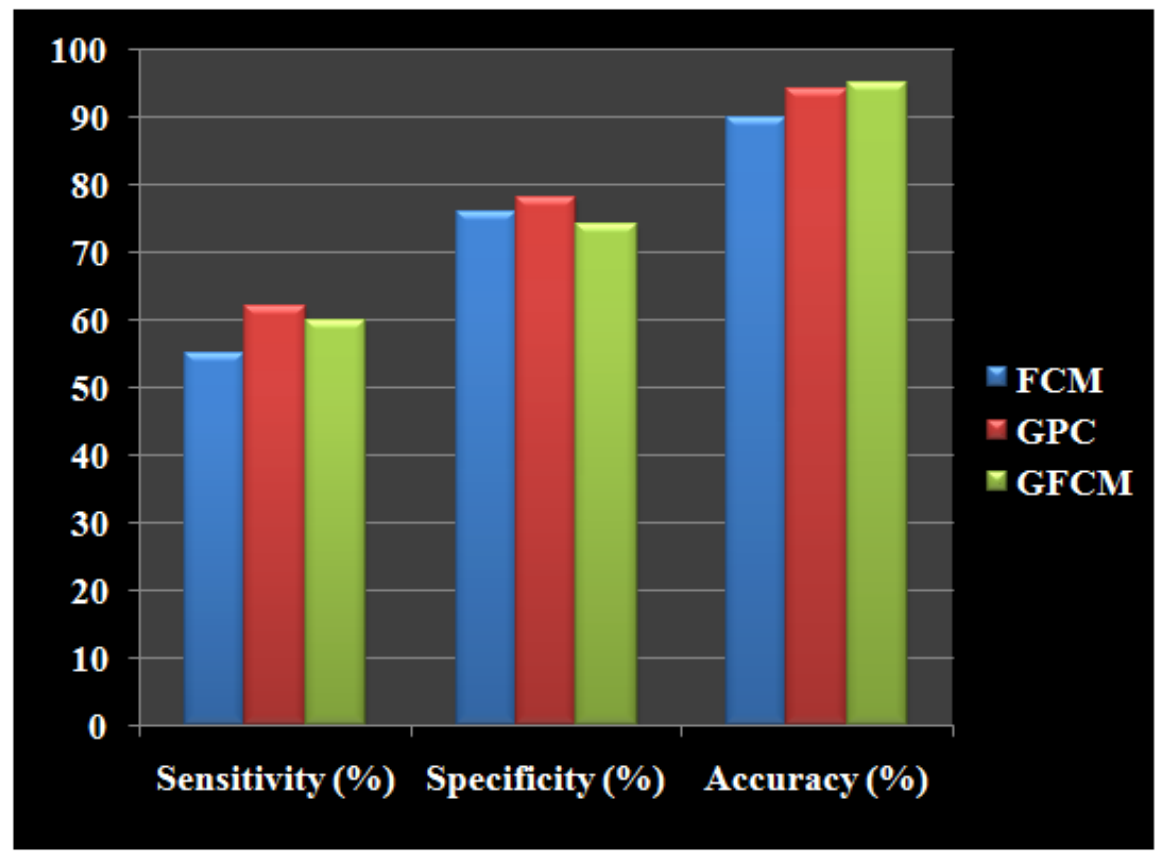

Figure 9. Comparing $S e, S p$ and $A c c$ of FCM, GPC and GFCM 
Computer Science \& Engineering: An International Journal (CSEIJ), Vol.2, No.2, April 2012

\section{CONCLUSION}

The proposed Fuzzy $c$-means clustering, Geostatistical Possibilistic clustering and Geostatistical Fuzzy c-means clustering methods are used for automatic detection of WMLs in brains of elderly people. The incorporation of the geostatistical estimate variance into the objective functions of fuzzy clustering and possibilistic clustering algorithms is relatively a simple and effective procedure for implementation and can be further explored using various advanced kriging systems in multivariate geostatistics. Experimental results using the MRI data of elderly individuals shows the advantages that Geostatistical Fuzzy c-means clustering is the best and effective approach for extracting White Matter Lesions. More accurate results are obtained by GFCM whereas GPC and FCM provide more false positives in brain image and they are less sensitive to noise. Experimental results over datasets show that GFCM is efficient and can reveal very encouraging results in terms of quality of solution found.

\section{REFERENCES}

[1] L. O. Wahlund, F. Barkhof, F. Fazekas, L. Bronge, M. Augustin,M. Sj”ogren, A. Wallin, H. Ader, D. Leys, L. Pantoni, F. Pasquier, T. Erkinjuntti, and P. Scheltens, "A new rating scale for age-related White Matter changes applicable to MRI and CT," Stroke, vol. 32, pp. 1318-1322, 2001.

[2] E. Matsusue, S. Sugihara, S. Fujii, E. Ohama, T. Kinoshita, and T. Ogawa, "White Matter changes in elderly people: MR-pathologic correlations,” Magn. Reson. Med. Sci., vol. 5, pp. 99-104, 2006.

[3] F. Fazekas, "Pathological correlates of incidental MRI White Matter signal hyper intensities," Neurology, vol. 43, pp. 1683-1689, 1993.

[4] P. Anbeek, K. L. Vincken, M. J. P. van Osch, R. H. C. Bisschops, and J. van der Grond, "Automatic segmentation of different-sized White Matter Lesions by voxel probability estimation," Med. Image Anal., vol. 8, pp. 205-215, 2004.

[5] R. Khayati, M. Vafadust, F. Towhidkhah, and S. M. Nabavi, "Fully automatic segmentation of multiple sclerosis lesions in brain MR FLAIR images using adaptive mixtures method and Markov random field model," Comput. Biol. Med., vol. 38, pp. 379-390, 2008.

[6] Z. Lao, D. Shen, D. Liu, A. F. Jawad, E. R. Melhem, L. J. Launer, R. N. Bryan, and C. Davatzikos, "Computer assisted segmentation of White Matter Lesions in 3D MR images using support vector machine,” Acad. Radiol., vol. 15, pp. 300-313, 2008.

[7] M. L. Seghier, A. Ramlackhansingh, J. Crinion, A. P Leff, and C. J. Price, "Lesion identification using unified segmentation-normalisation models and fuzzy clustering," NeuroImage, vol. 41, pp. 1253-1266, 2008.

[8] M. delC.V.Hernendez, K. J. Ferguson, F. M. Chappell, and J.M.Wardlaw, "Newmultispectral MRI data fusion technique for white matter lesion segmentation: method and comparison with thresholding in FLAIR images,” Eur. Radiol., Feb. 16, 2010.

[9] R. Beare, V. Srikanth, J. Chen, T. G. Phan, J. Stapleton, R. Lipshut, and D. Reutens, "Development and validation of morphological segmentation of age-related cerebral White Matter hyper intensities," NeuroImage, vol. 47, pp.199-203, 2009. 
Computer Science \& Engineering: An International Journal (CSEIJ), Vol.2, No.2, April 2012

\section{Authors}

Ms.M.Anitha received Bachelor of Engineering degree in Computer Science and Engineering in Avinasilingam University, Coimbatore in 2010. She is currently pursuing Master of Engineering degree in Computer Science and Engineering under Anna University, Chennai, India. Her areas of interest are Image Processing and Data Mining.

Prof.P.Tamije Selvy received B.Tech (CSE), Tech (CSE) in 1996 and 1998 respectively. Since 1999, she has been working as faculty in reputed Engineering Colleges. At Present, she is working as Assistant Professor (SG) in the department of Computer Science \& Engg, Sri Krishna College of Technology, Coimbatore. She is currently pursuing Ph.D under Anna University, Chennai. Her Research interests include Image Processing, Data Mining, Pattern Recognition and Artificial Intelligence.

Dr.V.Palanisamy received B.E (ECE), M.E (Communication Engg) and PhD (Communication Engg.) in 1972, 1974 and 1987 respectively. Since 1974, he has been the faculty of Electronics and Communication Engg. and Served at various Government engineering colleges. At present, he is the principal at Info Institute of Engineering, Coimbatore. His research interest is in the Heuristic search methods for Optimization problems in various applications. He is a Sr. member of ISTE, SIEEE, and CSI.
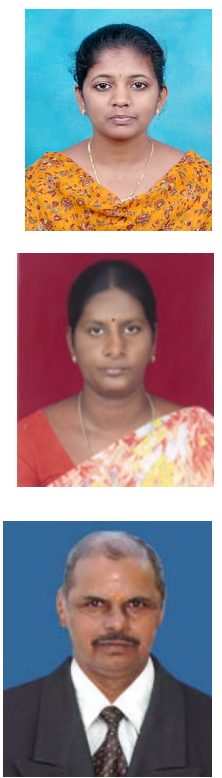